\title{
Die Vereinbarung zur Delegation ärztlicher Leistungen nach Anlage 24 BMV-Ä - Auswirkungen auf die Radiologie
}

Seit Jahren wird diskutiert, ob und in welchem Umfang es in der vertragsärztlichen Versorgung zulässig ist, dass der Radiologe Teile seiner Leistung auf nichtärztliches Personal delegiert. So ist bisher nicht geklärt, ob eine ausgebildete MTRA, welche in der Punktions- und Injektionstechnik geschult ist, Kontrastmittel auf Anweisung applizieren darf. Zwar regeln die $\S \S$ 15 Abs. 1 und 28 Abs. 1 SGB V, sowie $\S 15$ Abs. 1 S. 5 BMV-Ä, dass zur ärztlichen Behandlung die Hilfeleistungen anderer Personen gehören, die der Arzt anordnet und verantwortet. Gesetzliche Regelungen, die fachspezifische Delegationsmöglichkeiten festlegen, bestanden jedoch nicht, sodass sich die Bundesärztekammer und die Kassenärztliche Bundesvereinigung in einer Bekanntmachung zur persönlichen Leistungserbringung zuletzt am 29.08.2008 hierzu geäußert haben (vgl. Deutsches Ärzteblatt 2008, A 2173). Nun ist eine neue Vereinbarung zur Delegation ärztlicher Leistungen seit dem 01.10.2013 in Kraft getreten. Die Anlage 24 zum Bundesmantelvertrag-Ärzte (BMV-Ä) ist aus dem Auftrag des Gesetzgebers in $\S 28$ Abs. 1 S. 3 SGB V des GKVVersorgungsstrukturgesetzes vom 22.12.2011 (BGBl. I, S. 2983) an die Kassenärztliche Bundesvereinigung (KBV) und den GKV-Spitzenverband hervorgegangen. Danach sollten die Partner der Bundesmantelverträge bis zum 30. Juni 2012 für die ambulante Versorgung beispielhaft festlegen, bei welchen Tätigkeiten Personen ärztliche Leistungen erbringen können und welche Anforderungen an die Erbringung zu stellen sind.

Zunächst ist festzuhalten, dass der BMVÄ, nicht zuletzt aufgrund der beteiligten Vertragsparteien, nur für die vertragsärztliche Versorgung gilt (vgl. § 1 Abs. 1 Satz 1 BMV-Ä). Jedoch dürften insbesondere die getroffenen Regelungen für delegationsfähige Leistungen, Fernwirkungen auf andere Bereiche der medizinischen Versorgung wie der privatärztlichen Behandlung besitzen, da es auch in anderen Bereichen an konkreten Vorschriften mangelt (vgl. z. B. § 4 Abs. 2 GOÄ). In der Anlage 24 des BMV-Ä sind seit dem 1. Oktober 2013 beispielhafte, einer Delegation zugängliche, Leistungen aufgeführt. Damit ist die Anlage 24 des BMV-Ä als nicht abschließend zu betrachten, sodass für die in der Anlage nicht genannten Leistungen weiterhin die Delegationsfähigkeit im Einzelfall geprüft werden muss.

\section{Anforderungen an die Delegation in der Radiologie $\nabla$}

Im Anhang zur Anlage 24 des BMV-Ä werden in dem Beispielkatalog unter Punkt I. Nr. 4 die Durchführung technischer Untersuchungen in den Bereichen Röntgen, CT und MRT als delegierbare ärztliche Leistungen genannt. Dies entspricht der bisherigen Auffassung, wonach der Radiologe die Durchführung der technischen Untersuchung an entsprechend qualifizierte nichtärztliche Mitarbeiter delegieren kann. Dagegen obliegt die Anordnung der Leistung und die Befundung und Befundbewertung zwingend dem Radiologen.

In Bezug auf die Radiologie ergeben sich jedoch darüber hinaus, durch die Anwendung ionisierender Strahlung oder potenziell gefährlicher, für die Untersuchung notwendiger, Verabreichungen, zusätzliche Anforderungen. Entsprechende Vorgaben finden sich in den Leitlinien und Empfehlungen der Bundesärztekammer und der Qualitätssicherungsvereinbarungen der Kassenärztlichen Bundesvereinigung, den Richtlinien des Gemeinsamen Bundesausschusses, den Vorgaben der Strahlenschutzverordnung und der Röntgenverordnung (RöV) sowie der Richtlinie Fachkunde und Kenntnisse im Strahlenschutz bei dem Betrieb von Röntgeneinrichtungen in der (Zahn-) Medizin oder der Qualitätssicherungsrichtlinie zur Untersuchung und Behandlung von Menschen nach $\S \S 16,17$ RöV.

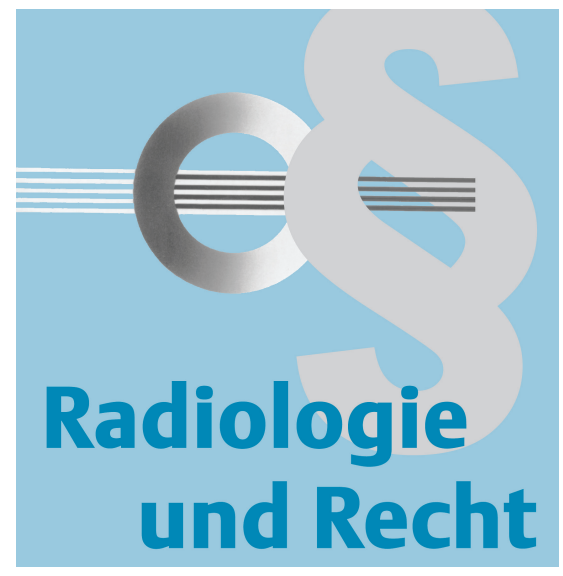

Am Beispiel der Röntgenverordnung wird deutlich, dass dort die Delegationsfähigkeit von Leistungen schon seit langem geregelt worden ist. Hierzu zählt insbesondere die Stellung der ,rechtfertigenden Indikation “ nach § 23 Abs. 1 RöV, die mit der Neufassung der RöV vom 18.06.2002 den Begriff der „ärztlichen Indikation“ ersetzt hat. Der Wegfall des Teilbegriffs „ärztliche“ führt jedoch keineswegs zu einer Delegationsfähigkeit der „rechtfertigenden Indikation“ an nichtärztliches Personal, da § 23 Abs. 1 Satz 5 RöV klarstellt, dass die rechtfertigende Indikation ausschließlich vom Arzt selbst gestellt werden muss. Die rechtfertigende Indikation umfasst gem. § 23 Abs. 1 Satz 2 RöV die positive Feststellung im Einzelfall, dass der gesundheitliche Nutzen der Anwendung von Röntgenstrahlen am Menschen gegenüber dem Strahlenrisiko überwiegt. Diese positive Feststellung muss auch bei einer Tätigkeit auf Überweisung, durch den die Strahlung anwendenden Arzt erfolgen, (vgl. § 23 Abs. 1 Satz 4 RöV). § 24 Abs. 2 RöV trifft insoweit Regelungen zur technischen Durchführung der Untersuchung. § 24 Abs. 2 Nr. 2 RöV stellt klar, dass MTRA berechtigt sind, ohne zusätzliche Fachkunde, die technische, eigenverantwortliche Durchführung vorzunehmen und dies sogar ohne ständige ärztliche Aufsicht. Lediglich eine allgemeine Aufsicht des Arztes muss bestehen.

In dem Anhang zur Anlage 24 des BMV-Ä wird nun unter Punkt I. Nr. 4. bestimmt, dass bei der Verwendung von Kontrastmitteln die Anwesenheit des Arztes erforderlich ist. Dies entspricht der bisherigen Auffassung, dass bei Röntgenuntersuchungen mit intravenöser Kontrastmittelgabe der Arzt wegen möglicher allergischer Reaktionen in unmittelbarer Nähe sein muss. Keine Feststellungen trifft der Anhang dagegen dazu, ob und in welchem 
Umfang bei allen anderen radiologischen Untersuchungsleistungen oder MRT-Untersuchungen, die nicht unter die RöV fallen, die Anwesenheit des Radiologen erforderlich ist. Hier dürfte weiterhin gelten, dass die Anwendung der Röntgenstrahlen als delegierte Leistung unter der Aufsicht und Verantwortung des fachkundigen Arztes zu erfolgen hat. Dabei ist zwar die Anwesenheit des Arztes im Röntgenraum nicht erforderlich, er muss jedoch für eventuelle Rückfragen der die Röntgenstrahlen anwendenden Mitarbeiter kurzfristig erreichbar sein und die auf seine Anordnung gefertigten Röntgenaufnahmen nach Erstellung selbst beurteilen, um daraus gegebenenfalls auch Schlussfolgerungen für ergänzende Aufnahmen ziehen zu können (vgl. Deutsches Ärzteblatt 2008, A 2173, 2176).

Für die vertragsärztliche Versorgung hat z.B. das LSG NRW bereits am 25.9.1996 (Az.: L 11 Ka 41/96 = NZS 1997, 195) entschieden, dass die Erbringung von Röntgenaufnahmen in einem von der Praxis 200 m entfernten Krankenhaus den Anforderungen an den Grundsatz der persönlichen Leistungserbringung nicht gerecht wird, wenn sich der Arzt nicht in den Räumlichkeiten aufhielt. Notwendig sei eine Überwachung des nichtärztlichen Personals einschließlich der jederzeitigen Möglichkeit des Arztes, eingreifen zu können. Dies sei ausgeschlossen, wenn z. B. in einer Filialsprechstunde in Abwesenheit des Arztes von nichtärztlichen Mitarbeitern radiologische Leistungen erbracht würden.

Diesen Umstand bildet nunmehr auch $\S 4$ der Anlage 24 BMV-Ä ab, der festlegt, dass der Vertragsarzt sicherzustellen hat, dass der Mitarbeiter aufgrund seiner beruflichen Qualifikation oder allgemeiner Fähigkeiten und Kenntnisse für die Erbringung der delegierten Leistung geeignet ist (Auswahlpflicht). Ferner hat der Arzt den Mitarbeiter zur selbständigen Durchführung der zu delegierenden Leistung anzuleiten (Anleitungspflicht) und diesen regelmäßig zu überwachen (Überwachungspflichten). Für den Umfang und die Intensität dieser ärztlichen Verpflichtungen ist die konkrete Qualifikation des Mitarbeiters ausschlaggebend. Generell bleibt es dabei, dass der jeweilige Arzt entscheidet, ob und an wen er eine Leistung delegiert (vgl. § 4 Abs. 1 Anlage 24 BMVÄ). Insoweit ist eine positive Entscheidung in jedem Einzelfall notwendig, um die Delegation einer ärztlichen Leistung vorzu- nehmen. Die Berechtigung gem. § 24 Abs. 2 Nr. 2 RöV, eine ausgebildete MTRA einzusetzen, korrespondiert mit den Regelungen der $\S \S 9,10$ MTAG. Gem. $§ 9$ Abs. 1 Nr. 2 MTAG bestehen Tätigkeitsbereiche, die zweifelsfrei an MTRA delegationsfähig sind. Hierzu gehören die Durchführung der technischen Arbeiten und Beurteilung ihrer Qualität in der radiologischen Diagnostik und anderen bildgebenden Verfahren einschließlich der Qualitätssicherung, die technische Mitwirkung in der Strahlentherapie bei der Erstellung des Bestrahlungsplanes und dessen Reproduktion am Patienten einschließlich der Qualitätssicherung, der technischen Mitwirkung in der nuklearmedizinischen Diagnostik und Therapie einschließlich der Qualitätssicherung und der Durchführung messtechnischer Aufgaben in der Dosimetrie und im Strahlenschutz in der Radiologischen Diagnostik, der Strahlentherapie und der Nuklearmedizin.

Dieser gesamte Regelungsgehalt, inklusive der ausdrücklich vorgesehenen Möglichkeiten der Delegation, war nach den Regelungen der RöV bereits vor dem in Krafttreten der Anlage 24 BMV-Ä wirksam, sodass der Regelungsgehalt, bezogen auf die Radiologie, einen überwiegend klarstellenden Charakter besitzt.

\section{Delegation in einer Gemein- schaftspraxis \\ $\nabla$}

Hinsichtlich der Frage, ob und in welchem Umfang Leistungen als persönlich erbracht angesehen werden können, die von anderen Ärzte erbracht worden sind, ist auch auf die Praxisform und das betreffende ärztliche Fachgebiet abzustellen.

Wie bei den in $\S 15$ Abs. 1 BMV-Ä aufgeführten angestellten Ärzten oder Praxisassistenten, erfolgt auch eine Zurechnung der Leistungen der Ärzte untereinander in einer fachgleichen Gemeinschaftspraxis. Steinhilper (Lexikon des Arztrechts, 4060 „Persönliche Leistungserbringung“ Rdnr. $61 \mathrm{ff}$.) führt hierzu in diesem Sinne aus:

„Handelt es sich um eine Einzelpraxis, darf zur selben Zeit in der Vertragsarztpraxis („Stammsitz“) keine ärztliche Leistung (von Hilfspersonal) erbracht werden. Etwas anderes gilt nur, wenn in einer (fachgleichen) Gemeinschaftspraxis der eine Partner an der einen, der andere an der anderen Stelle die fachliche Aufsicht vor Ort übernimmt.“
Das bedeutet, dass die fachliche Aufsicht über die Erbringung von ärztlichen Leistungen in einer Gemeinschaftspraxis für einen Praxispartner auch in dessen Abwesenheit durch die übrigen Gesellschafter ausgeübt werden kann. Die Voraussetzungen hierfür sind:

- Fachgebietsidentität,

- gleicher Aus- und Weiterbildungsstand,

- gleiche Abrechnungsgenehmigungen bei besonderen Untersuchungs- und Behandlungsmethoden in der GKV nach $\S 135$ Abs. 2 SGB V.

$\mathrm{Zu}$ den Abrechnungsgenehmigungen im Bereich der Radiologie, die in der vertragsärztlichen Versorgung für bestimmte Leistungen vorliegen müssen, gehören insbesondere die „Vereinbarung zur Strahlendiagnostik und -therapie“, die Kernspintomografie-Vereinbarung und die Qualitätssicherungsvereinbarung zur interventionellen Radiologie.

Soweit daher während der Abwesenheit eines Arztes in der Praxis ständig mindestens ein Facharzt für Radiologie mit der entsprechenden Fachkunde anwesend ist, ist davon auszugehen, dass die MTRA, die die Untersuchungen durchgeführt haben, ausreichend angeleitet und überwacht werden. Die Frage ist allerdings, ob vertragsärztlich gefordert werden kann, dass es sich bei dem anwesenden Partner der Gemeinschaftspraxis um einen Vertragsarzt handelt, wie dies von manchen KVen gefordert wird.

Daneben ist zu berücksichtigen, dass der BMV-Ä bei sog. gerätebezogene Untersuchungsleistungen Lockerungen vom Grundsatz der persönlichen Leistungserbringung vorsieht. Nach $\S 15$ Abs. 3 BMVÄ können sich Ärzte bezogen auf diese Leistungen zu einer sog. Leistungserbringungsgemeinschaft zusammenschließen, die es erlaubt, dass auch bei Abwesenheit eines Arztes, ein anderer Arzt, der über die gleichen Qualifikationsvoraussetzungen verfügt, diese Leistungen erbringen darf.

$\S 15$ Abs. 3 BMV-Ä hat folgenden Wortlaut:

„(3) Vertragsärzte können sich bei gerätebezogenen Untersuchungsleistungen zur gemeinschaftlichen Leistungserbringung mit der Maßgabe zusammenschließen, dass die ärztlichen Untersuchungsleistungen nach fachlicher Weisung durch einen der 
beteiligten Ärzte persönlich in seiner Praxis oder in einer gemeinsamen Einrichtung durch einen gemeinschaftlich beschäftigten angestellten Arzt nach $\S 32$ b Ärzte-ZV erbracht werden. Die Leistungen sind persönliche Leistungen des jeweils anweisenden Arztes, der an der Leistungsgemeinschaft beteiligt ist. Sind Qualifikationsvoraussetzungen gemäß $\S 11$ dieses Vertrages vorgeschrieben, so müssen alle Gemeinschaftspartner und ein angestellter Arzt nach $\S 32$ b Ärzte-ZV, sofern er mit der Ausführung der Untersuchungsmaß-nahmen beauftragt ist, diese Voraussetzungen erfüllen.“

$\S 15$ Abs. 3 BMV-Ä erlaubt bei gerätebezogenen Untersuchungsleistungen, dass die ärztlichen Untersuchungsleistungen nach fachlicher Weisung durch einen der beteiligten Ärzte persönlich in seiner Praxis oder in einer gemeinsamen Einrichtung durch einen gemeinschaftlich beschäftigten angestellten Arzt nach $\S 32$ b Ärzte-ZV erbracht werden können. Die Leistungen sind persönliche Leistungen des jeweils anweisenden Arztes, der an der Leistungsgemeinschaft beteiligt ist. Röntgen-, CTund MRT-Leistungen dürften unstreitig solche gerätebezogene Leistungen sein.

In der Kommentierung von Steinhilper (Lexikon des Arztrechts, 4060 „Persönliche Leistungserbringung“ Rdnr. 35) heißt es hierzu:

„§ 15 Abs. 3 BMV-Ä [...] und $\S 14$ Abs. 2 BMV-Ersatzkassen [...] brachten unter dem Gesichtspunkt der Wirtschaftlichkeit und Rationalisierung eine erhebliche Erleichterung für die Ärzte. Musste bisher ein Vertragsarzt mit dem Patienten jeweils zu einem gemeinschaftlich angeschafften Gerät, um dort die Geräteleistung selbst durchführen und/oder überwachen zu können, so kann seither innerhalb eines Ärztepools einer die Untersuchung für die an der $\mathrm{Ge}$ meinschaft beteiligten anderen Ärzte übernehmen; die jeweils anweisenden Ärzte dürfen diese Leistungen als persönlich erbrachte Leistungen abrechnen.“

Nach dem Rundschreiben der Kassenärztlichen Bundesvereinigung vom10.04.1995 sind die Formen der Zusammenarbeit zwischen Ärzten einer Leistungserbringungsgemeinschaft sehr vielschichtig; u. a. ist eine Leistungserbringung in einem ausgelagerten Praxisteil möglich.

Die Vorschrift des $\S 15$ Abs. 3 BMV-Ä belegt, dass gerade bei gerätebezogenen Un- tersuchungsleistungen, zu denen radiologische Leistungen unstreitig gehören, eine ausnahmslose Anwesenheitspflicht aller Ärzte einer Gemeinschaftspraxis nicht besteht. Insbesondere ist es zulässig, wenn die übrigen Ärzte die Überwachung der Leistungserbringung durch die MTRA übernehmen.

\section{Kontrastmittelinjektion durch MTRA \\ $\nabla$}

Die Delegationsmöglichkeiten bzgl. der Kontrastmittelinjektion durch MTRA sind in der Radiologie noch nicht zweifelsfrei geklärt. Auch hier muss die Prüfung und Anordnung der Delegationsfähigkeit der jeweiligen Leistungen in jedem Einzelfall erfolgen. Zudem muss der Arzt den Schwierigkeitsgrad und das Risiko anhand des zu ermittelnden Gesamtzustandes abwägen und positiv feststellen, dass in diesem konkreten Einzelfall eine Delegation möglich erscheint. Des Weiteren muss die Delegation schriftlich festgehalten werden und vom Arzt persönlich gegengezeichnet werden. Die jeweilige Dokumentation muss den Patienten, sofern diese nicht in die Patientenakte aufgenommen wird, namentlich kennzeichnen und die zu verabreichenden Medikamente in Menge, Art und Zeitpunkt genau bestimmen.

Hinzu tritt, dass die mit der delegierten Aufgabe betraute MTRA, mittels ihrer spezifischen persönlichen Voraussetzungen, die Leistung unzweifelhaft erbringen kann. Im Falle der Injektion von Kontrastmitteln gehört hierzu die erworbene Qualifikation in der Punktions- und Injektionstechnik, die von dem jeweiligen Fachvorgesetzten zuvor geprüft und schriftlich bestätigt wurde. Überwachungs- und Beaufsichtigungspflichten bleiben hiervon unberührt. Eine weitere Voraussetzung ist, dass sämtliche nicht delegierbaren Leistungen vom Arzt persönlich und ordnungsgemäß erbracht und dokumentiert wurden und der Patient seine umfassende Einwilligung in die Behandlung erteilt hat. Zur Aufklärung des Patienten gehört hierbei nicht der Umstand der etwaig erfolgenden Delegation von Leistungen, sodass der Patient in dem Gespräch nicht darüber aufgeklärt werden muss, dass zunächst dem Arzt zugeschriebene Leistungsanteile auf nichtärztliche Mitarbeiter zulässig unter Aufsicht delegiert werden (so auch bereits OLG Stuttgart vom 01.12.1994 Az.:14 U 48/93). Insoweit besteht, nicht zuletzt aufgrund des Urteils des OLG Dresden vom 24.07.2008 (Az.: 4 U 1857/07), seit geraumer Zeit die Möglichkeit, Kontrastmittelapplikationen auf eine geschulte MTRA zu übertragen. Das OLG Dresden stellt in dem Urteil von 2008 fest, dass eine MTRA in einer radiologischen Großpraxis auch generell berechtigt ist, unter Aufsicht des verantwortlichen Arztes intravenöse Injektionen mit, im befassten Fall, schwach radioaktivem Technetium vorzunehmen. Zwar stelle eine derartige Injektion einen Eingriff dar, der zum Verantwortungsbereich des Arztes gehört. Es handele sich hierbei aber nicht um eine Tätigkeit, die aufgrund ihrer Schwierigkeit, Gefährlichkeit oder Unvorhersehbarkeit zwingend von einem Arzt erbracht werden muss.

Auch die Anlage 24 BMV-Ä geht in dem Anhang unter Punkt I. Nr. 4 davon aus, dass die Kontrastmittelinjektion unter bestimmten Voraussetzungen delegierbar ist, da als fachlich geeignete Mitarbeiter folgende Personen benannt werden:

- Medizinische / $\mathrm{r}$ Fachangestellte / $\mathrm{r}$ (MFA) [Röntgen: Fortbildung „Strahlenschutzgemäß § 24 Abs. 2 Nr. 4 RöV“]

- Medizinisch-Technische/r Assistent/in (MTA) [Fachkenntnisse gemäß Strahlenschutzverordnung]

- Medizinisch-technische/ $\mathrm{r}$ Radiologieassistent/-in (MTRA) [Fachkenntnisse gemäß Strahlenschutzverordnung].

Während Punkt I. Nr. 4 lediglich die Anwesenheit eines Arztes bei der Kontrastmittelinjektion vorschreibt, bestimmt Punkt II Nr. 8, dass die Injektion von Radionukliden ausschließlich durch den Arzt selbst zu erfolgen hat.

\section{Auswirkungen auf die Radiologie $\nabla$}

Im Ergebnis ist festzuhalten, dass die konkreten Auswirkungen der Regelungen in der Vereinbarung auf die Radiologie nicht übermäßig groß sind, da rechtlichen Anforderungen schon weitgehend in der RöV geregelt sind. Zu begrüßen ist allerdings, dass jetzt eine Rechtsgrundlage für die Zulässigkeit der Applikation von Kontrastmitteln durch MTRA besteht, auch wenn dies bisher schon durch die Rechtsprechung anerkannt worden ist.

Es fehlt aber nach wie vor in wesentlichen Bereichen an einer Klarstellung bzgl. der 
Anforderungen an die persönliche Leistungserbringung im Vertragsarztrecht. Dies gilt umso mehr, als die Rechtsprechung davon ausgeht, dass die einschlägigen Rechtsnormen des Vertragsarztrechts gegenüber denen der RöV spezielleren Charakter haben und es daher insbesondere nach den Vorgaben der Abrechnungsbestimmungen des EBM und des HVM darauf ankommt, „ob der Vertragsarzt befugt ist, diese Leistung zur selbständigen Erbringung auf MTAs zu übertragen“. Danach ist weiterhin davon auszugehen, dass der Vertragsarzt Leistungen der MTRA weiterhin nur abrechnen darf, wenn diese Leistungen unter seiner Aufsicht in seinem unmittelbaren Einflussbereich erbracht worden sind (vgl. LSG NRW, Urt. v. 25.9.1996, Az.: L 11 Ka 41/96 = NZS 1997, 195, 197). Zu beachten ist, dass Verstöße gegen die vertragsarztrechtlichen Abrechnungsbestimmungen die KV zur Rückforderung des Honorars berechtigt, sodass die Anforderungen sehr ernst genommen werden müssen. Hier hätte man sich gewünscht, dass die Vereinbarung die bestehenden Unklarheiten beseitigt hätte. Im Ergebnis bleibt daher festzuhalten, dass die Anlage 24 BMV-Ä inkl. des Anhanges im Bereich der Radiologie zwar eine klarstellende Funktion besitzt, ohne jedoch tatsächlich etwas Neues zu regeln.

Jens Remmert, LL.M.

Rechtsanwalt

Dr. Peter Wigge

Rechtsanwalt

Fachanwalt für Medizinrecht

Rechtsanwälte Wigge

Scharnhorststr. 40

48151 Münster

Telefon: (0251) 53595-0

Telefax: (0251) 53595-99

E-Mail: kanzlei@ra-wigge.de

www.ra-wigge.de 